For Publisher's use

\title{
SUMMARY OF ICHEP 2004
}

\author{
JOHN ELLIS \\ Theory Division, CERN, CH-1211 Geneva 23, Switzerland
}

\begin{abstract}
Aspects of ICHEP 2004 are summarized from a theoretical point of view. QCD works, new NNLO calculations are becoming available and new string calculational tools are emerging, but no conclusions can yet be drawn about the discovery of the quark-gluon plasma or pentaquarks. The small upward shift in the experimental value of $m_{t}$ raises somewhat the central value of the Higgs mass extracted from a global electroweak fit, and the CKM model describes well the data from the B factories. The SuperKamiokande, KamLAND and K2K experiments have evidence for oscillation dips in their neutrino data. Little Higgs models are interesting alternatives to low-energy supersymmetry for stabilizing the electroweak scale. Convincing experimental evidence for dark matter particles is still lacking. The LHC is on its way, the technology choice clarifies the roadmap for the ILC, and a multi-TeV CLIC would also have rich physics agenda.
\end{abstract}

hep-ph/0409360

\section{Introduction}

Summarizing this conference with its wealth of new results is an interesting challenge, and I apologize in advance to those whose work I neglect or under-emphasize.

A good place to start my task is the basic Lagrangian underlying particle physics ${ }^{1}$ :

$$
\begin{aligned}
\mathcal{L} & =-\frac{1}{4} F_{\mu \nu}^{a}+i \bar{\psi} \not D \psi+\psi_{i} \lambda_{i j} \psi_{j} h+h . c . \\
& +\left|D_{\mu} h\right|^{2}-V(h) \\
& +\left(\frac{1}{M} L_{i} \lambda_{i j}^{\nu} L_{j} h^{2} \text { and } / \text { or } L_{i} \lambda_{i j}^{\nu} N_{j} h+h . c .\right)
\end{aligned}
$$

The first two terms constitute the gauge sector of the theory, the next two terms describe quark flavour physics, the next two are alternative and complementary contributions to neutrino masses, and the last two terms represent the electroweak symmetry-breaking sector of the Standard Model. The following sections of this talk discuss these various sectors in turn. Subsequently, sections are devoted respectively to string theory, the connection between particle physics and cosmology, and the prospects for future accelerators. Finally, this conference summary is itself summarized by a few concluding remarks.
CERN-PH-TH/2004-167

\section{The Gauge Sector}

\section{$2.1 Q C D$}

The challenge in QCD is no longer to test the theory, but rather to understand and calculate it better ${ }^{2}$. Perturbative calculations are most reliable in the high-energy regime, where they provide the essential baselines for searches for new physics beyond the Standard Model. The high temperatures and pressures attained in relativistic heavy-ion collisions may eventually offer us another relatively simple playground, and the lattice is an increasingly accurate tool for non-perturbative calculations, but hadron spectroscopy does not cease to pose important challenges to our qualitative understanding of QCD.

As we heard here, the latest data from both the FNAL Tevatron ${ }^{3}$ and HERA ${ }^{4}$ at DESY are in good agreement with perturbative QCD calculations. This is true, in particular, for the total jet cross sections (after adjusting the parton distribution functions) and for heavy-flavour production. In the latter case, previous discrepancies between theory and experiment have dissipated with the advent of new calculations ${ }^{5}$ and measurements ${ }^{3,4}$. This is reassuring for the LHC, which will depend on Tevatron and HERA inputs in its searches for new physics. Several 
new measurements of $\alpha_{s}$ were reported here, in particular from HERA ${ }^{4}$. The current world average value of $\alpha_{s}\left(m_{Z}\right)$ is $0.1182 \pm$ $0.0027^{6}$, which (reassuringly) has changed little from the corresponding average a couple of years ago.

Significant progress has been reported here in the drive towards greater precision in QCD calculations ${ }^{2}$. The full NNLO expressions for the quark and gluon splitting functions are now known ${ }^{7}$, and cover several pages! They lie comfortably within the range expected from previous incomplete calculations. They open the way to a new era of high-precision QCD, which has already been inaugurated by comparisons of $\mathrm{W}$ and $\mathrm{Z}$ production data from the Tevatron collider with NNLO cross sections ${ }^{2}$ - these might provide a useful tool for measuring the LHC luminosity. Important parts of the NNLO calculations of hadronic jet cross sections have also been completed, and are likely to be complete by the time the LHC is switched on, though there are still important technical issues in the infra-red cancellations needed for the NNLO calculations for multi-jet production.

A very exciting theoretical development has been the proposal of a new and powerful string approach to QCD calculations ${ }^{8}$. It uses the simple amplitudes for maximal helicity-violating (MHV) multi-gluon processes ${ }^{9}$ as effective vertices in a new graphical approach based on scalar field theory. The MHV amplitudes are combined with scalar propagators to calculate tree-level non-MHV amplitudes for both quarks and gluons, as illustrated in Fig. 1, and may also be used to calculate loop diagrams ${ }^{8}$. This method is dramatically simpler than conventional techniques, providing compact outputs expressed in terms of familiar spinor products. There are high hopes of using this technique for phenomenology ${ }^{10}$, with calculations of multijet cross sections underway for the LHC.

Another 'hot' topic at the moment is the

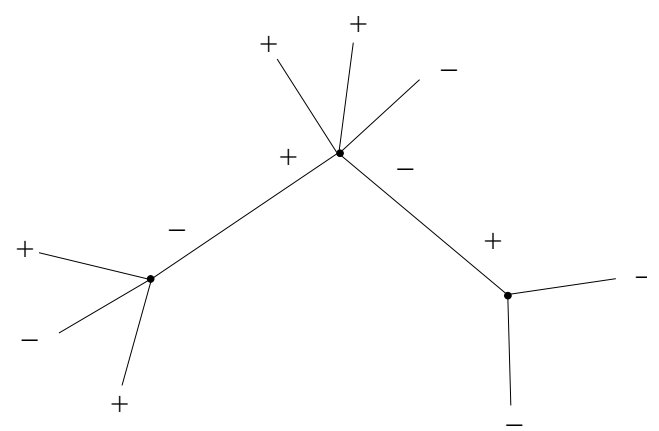

Figure 1. Illustration how MHV multigluon amplitudes can be combined to yield tree-level non-MHV amplitudes $^{8}$. The \pm signs indicate the gluon helicities.

QCD phase diagram, which is expected to exhibit a colour-superconductor phase at high baryon density and a quark-gluon-plasma (QGP) phase at high temperatures, that may be explored in relativistic heavy-ion collisions ${ }^{11}$. These phases are not expected to be separated by a strong first-order phase transition, though there may be an interesting critical point to explore, if one can devise observable signatures of it. Thermodynamics should allow reliable calculations to be made for the QGP phase, but our ability to confront them with experiment is hampered by the facts that thermalization may not be complete, that the QGP is expected to be strongly-interacting ${ }^{12}$, and that many probes involve hadrons produced after the QGP has cooled. Nevertheless, the measured abundances of the many particle species are well described by a simple thermodynamic model, with a freeze-out temperature $T$ similar to that predicted by lattice calculations, and a baryonic chemical potential that decreases with increasing collision energy. However, is it possible that these abundances may just be reflecting phase space and statistics?

Considerable interest has arisen from RHIC measurements of the elliptic flow variable $v_{2}{ }^{11}$, which reflects the shape of the interaction region. In peripheral collisions, this is expected to start with an almond shape. 
It should then expand more rapidly in the direction of the minor axis, where the pressure is greater, so that the shape becomes more spherical at later times as the interaction region cools. This expected behaviour is seen in the transverse momentum spectra, which exhibit higher $v_{2}$ for the higher- $p_{T}$ particles that are presumably more likely to have been produced earlier. Moreover, the elliptic flows for different hadrons scale approximately with the numbers of quarks they contain,

$$
v_{2}^{M}\left(p_{T}\right) \sim 2 v_{2}^{q}\left(p_{T} / 2\right), v_{2}^{B}\left(p_{T}\right) \sim 3 v_{2}^{q}\left(p_{T} / 3\right),
$$

in agreement with the idea that the values of $v_{2}$ originate with their constituents during a QGP phase. The measurements are reproduced well by a hydrodynamic model using a simplified quark-gluon equation of state.

However, simple hydrodynamic models fail to reproduce the source size inferred from HBT interference measurements ${ }^{11}$. If some quark-gluon fluid is being formed, it seems to resemble a liquid rather than an ideal gas, and complications such as strong interactions and viscosity may need to be incorporated in modelling it.

The poster-child for QGP production at RHIC may be the observation of jet quenching ${ }^{11}$, illustrated in Fig. ${ }^{13}$. This occurs in central $A u+A u$ (but not $D+A u$ or minimumbias $p+p)$ collisions on the side opposite a transverse jet, and is stronger out of the overall event plane, where the initial density is thought to be higher. The QGP interpretation is that the opposite-side parton loses its transverse energy in collisions with coloured partons in the QGP. This jet quenching is accompanied by an increased $\gamma / \pi^{0}$ ratio at larger $p_{T}$, and softening of the jet fragmentation function.

Are these observations, as well as others including the enhancement of low-mass pairs and the suppression of $J / \psi$ particles observed in earlier relativistic heavy-ion collisions at the CERN SPS, sufficient to claim

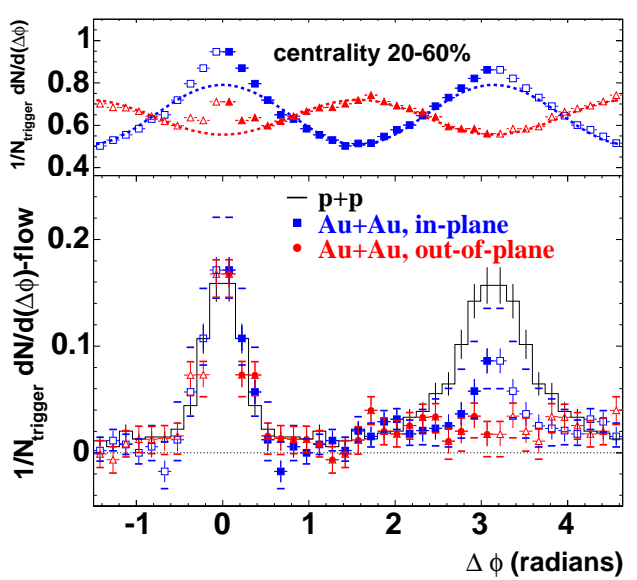

Figure 2. The azimuthal distributions of high- $p_{T}$ particles in $A u+A u$ collisions at RHIC (bottom panel) differ from the standard pattern of elliptic flow (top panel) ${ }^{13}$. The suppression compared with $p+p$ collisions (shown) and $D+A u$ collisions (not shown) is interpreted as the quenching of jets by finalstate interactions in a hot and dense medium. The opposite-side suppression is greater out of the reaction plane than in it, as expected because the pressure and density should be greater in that direction ${ }^{11}$.

discovery of the expected QGP? RHIC data are still described by a patchwork of theoretical approaches, with model parameters often adjusted independently for different observables. Moreover, some observables, such as particle ratios, may have alternative explanations, and others, such as the HBT radii are not yet reproduced well by simple QGP calculations. The parton energy loss apparently observed in jet quenching is a very promising development, and it would be interesting to hear from RHIC about the $J / \psi$ suppression already observed at the CERN SPS. In the absence of a 'smoking gun' for a compelling QGP claim, I share the view of the rapporteur here ${ }^{11}$ that we still need more quantitative estimates of the theoretical uncertainties.

The final QCD topic to discuss is hadron spectroscopy, which is experiencing an ex- 
perimental renaissance at the moment 14 . There has been much discussion here whether pentaquarks or other exotic hadrons exist. The quark descriptions of the $D_{s J}(2317)$ and $D_{s J}(2460)$ have also been debated ${ }^{15}$, as has the existence of the $D_{s J}(2632)$ state reported by SELEX ${ }^{16}$. The quark description of the $X(3872)$ discovered by BELLE is also intriguing, as is the fate of the $12 \%$ rule in $\psi^{\prime}$ decay investigated by BES and the interpretations of other threshold states they have reported here. In the time available, I shall concentrate on pentaquarks, the $X(3872)$ and some remarks about threshold states.

Sightings of the $\Theta^{+}(1540)$ pentaquark candidate were reported here by HERMES ${ }^{17}$ and ZEUS ${ }^{18}$, as well as the observation of a $\Theta_{c}(3095)$ by $\mathrm{H} 1{ }^{19}$. In addition to these, there have also been many other observations of the $\Theta^{+}(1540)^{14}$, and one report of an exotic $\Xi^{--}(1862)$ baryon ${ }^{20}$. One puzzle in the $\Theta^{+}(1540)$ observations has been that the masses and decay widths have varied between experiments ${ }^{21}$. Generally speaking, observations of the $\Theta^{+}(1540)$ in the $n K^{+}$final state tend to give higher masses than observations in the $p K_{s}^{0}$ final state, as seen in Fig. 3. Also, the two positive experiments reporting observations here yield non-zero decay widths: $\Gamma_{\theta}=17 \pm 9 \pm 2 \mathrm{MeV}^{17}$ and $\Gamma_{\theta}=8 \pm 4 \mathrm{MeV}^{18}$, whereas partial-wave analyses of $K^{+} N$ scattering indicate an upper limit $\Gamma_{\theta}<1 \mathrm{MeV}^{23}$.

Several other experiments (including a number reported here) have not seen these candidate pentaquark states ${ }^{14}$. How can one assess the significance of these negative experiments? One strategy is to compare the obtained upper limit with the trend line of the measured rates for the production of other, well-established baryons. The (upper bound on the) yield of the $\Theta^{+}(1540)$ is often compared with that of the $\Lambda(1520)$. However, as discussed here by the BABAR collaboration ${ }^{24}$, the $\Lambda(1520)$ yield is anomalously high, compared with trend line. Nevertheless, the upper bound on $\Theta^{+}(1540)$ produc-

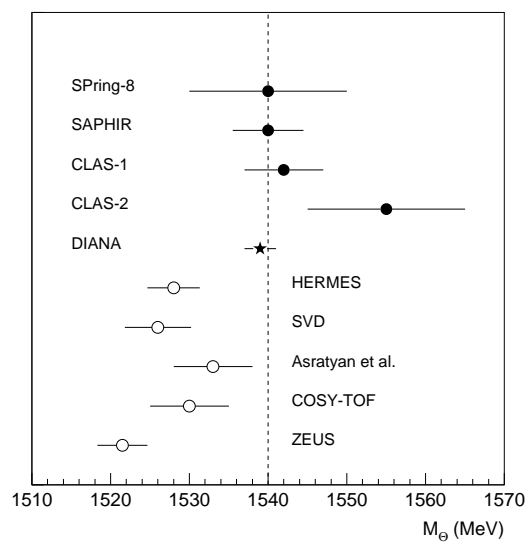

Figure 3. The mass of the $\Theta^{+}(1540)$ measured in $n K^{+}$final states (solid circles) tends to be larger than in $p K_{s}^{0}$ final states (open circles) $22,21,14$. The starred measurement was made in an experiment connecting $n K^{+} \rightarrow p K_{s}^{0}$.

tion is significantly lower than this trend line, as is the BABAR upper limit on $\Xi^{--}(1862)$ production. Generally speaking, most positive results for $\Theta^{+}(1540)$ are at relatively low energies, and not at high energies ${ }^{25}$, suggesting that exotic baryons may have production mechanisms that are different from those of conventional baryons. For example, their photo- and electroproduction may be facilitated by the $\bar{s} s$ component of the photon.

The interpretation of the $\Theta^{+}(1540)$ could be very interesting ${ }^{21,26}$ — if it exists! In order to accommodate it, naïve non-relativistic quark models would need epicycles such as dior triquarks 27,28 , and the ground state would involve some $P$-wave configuration, an unfamiliar option within the quark model. However, something very much like the $\Theta^{+}(1540)$, in both mass and narrow width, was predicted ${ }^{29}$ in the chiral soliton model ${ }^{30}$, which is based on the idea that quarks are intrinsically very light - a few $\mathrm{MeV}$ for the up and down and $\sim 100 \mathrm{MeV}$ for the strange - and that baryons are topological clouds effectively composed of very many quarks. In addition 
to fitting the data on the $\Theta^{+}(1540)$ and the $\Xi^{--}$(1862), the chiral soliton model also predicts other exotic states, filling out an antidecuplet of $S U(3)$, as well as other nearby $27-$ and 35 -dimensional representations ${ }^{31}$.

The existence of the $\Theta^{+}(1540)$ still lacks conclusive confirmation - even a single highstatistics, high-significance experiment would suffice. If it does exist, measurements of the $\Theta^{+}$spin and parity would distinguish between the chiral soliton and rival quark models. The stakes are high: if they exist, the $\Theta^{+}, \Xi^{--}$and $\Theta_{c}$ may take us beyond the naïve quark model.

The next spectroscopic surprise that I discuss is the $X(3872)$ state ${ }^{14}$ discovered by Belle ${ }^{32}$ and confirmed by $\mathrm{CDF}^{33}$ and BABAR ${ }^{34}$. Here the theoretical debate is whether it is a (displaced) charmonium state, which would need unnatural spin-parity such as $1^{++}$in order to avert rapid decay into $D \bar{D}$, or, in view of its near-degeneracy with the $D^{0} D^{* 0}$ threshold, whether it should better be regarded as a $D^{0} D^{* 0}$ 'molecular state' ${ }^{21}$. The suspicion is that the $\pi \pi$ pair in the $\pi \pi J / \psi$ mode first observed may be in a $\rho$ state, in which case the recent Belle report of $X(3872) \rightarrow \omega J / \psi$ decay ${ }^{35}$ would be very interesting. It would be evidence for isospin violation in $X(3872)$ decay, which would be a signature of a $D^{0} D^{* 0}$ admixture in its wave function.

There have been several other reports here of hadronic threshold states, from BES ${ }^{36}$ and Belle ${ }^{38}$ in particular. The most impressive is the $\bar{p} p$ state with a mass of $M=$ $1859_{-10-25}^{+3+5} \mathrm{MeV}$ and a decay width $\Gamma<30$ $\mathrm{MeV}^{36}$, but other states have also been reported in the $\bar{p} \Lambda, \bar{p} \Lambda_{c}, \bar{K} \Lambda, \pi \pi^{37}$ and $\pi K$ combinations ${ }^{14}$. These might be candidates for tetra- or even sextaquark states, but an alternative would be that the quark description is not optimal for discussing such nonrelativistic hadronic combinations ${ }^{21}$. Perhaps they should rather be described in terms of hadronic physics in a first approximation, as are nuclei.

\subsection{Electroweak Physics}

The electroweak sector of the Standard Model continues to withstand experimental attacks ${ }^{39}$, despite some points of hot discussion such as the comparison between low- and high-energy data. Measurements of parity violation in atomic physics and Møller scattering are compatible with the expected running of $\alpha_{e m}\left(Q^{2}\right)$, but the NuTeV measurement of $\sin _{W}^{2}$ is offset by about $3 \sigma^{40}$. Improved electroweak radiative corrections as well as an asymmetry in the strange sea and a difference: $\bar{u} \neq \bar{d}$ might each account for about $1 \sigma$ of this discrepancy ${ }^{39}$. Light will be cast on the NuTeV measurement of $\sin _{W}^{2}$ by the forthcoming NOMAD result ${ }^{41}$, so I follow the rapporteur ${ }^{39}$ in leaving $\mathrm{NuTeV}$ out of the global electroweak fit for the time being.

The big news in the electroweak sector has been the new value of the top-quark mass reported by $\mathrm{D} \varnothing{ }^{42}$, on the basis of a reanalysis of their Run 1 data: $m_{t}=179.0 \pm 5.1$ $\mathrm{GeV}$ which, when combined with the previous CDF measurement, yields a world average $m_{t}=178.0 \pm 4.3 \mathrm{GeV}$. There are good prospects for further reducing the experimental error using Run 2 data from both $\mathrm{D} \varnothing$ and $\mathrm{CDF}^{43}$.

The $\mathrm{D} \varnothing$ result is important news, in particular because of its implications for the Higgs mass predicted in the global electroweak fit ${ }^{39,44}$. The new value of $m_{t}$ increases the prediction for $m_{H}$ by $\sim 20 \mathrm{GeV}$ and new 2-loop terms in $m_{W}$ and $\sin _{W}^{2}{ }^{45}$, as well as other theoretical improvements, increase $m_{H}$ by $\sim 6 \mathrm{GeV}$, yielding

$$
m_{H}=114_{-45}^{+69} \quad \mathrm{GeV}
$$

as the present best estimate using an experiment-driven ${ }^{46}$ value of $\alpha_{e m}\left(m_{Z}\right)$ : see Fig. 4 . The central value of $m_{H}$ would be increased by $\sim 15 \mathrm{GeV}$ if a theory-driven value of $\alpha_{e m}\left(m_{Z}\right)$ with a smaller error were chosen ${ }^{39}$. The largest pull on the global elec- 
troweak fit comes from the forward-backward asymmetry for $b$ quarks, and heavy-flavour measurements of $\sin _{W}^{2}$ tend to yield values different from leptonic measurements and $m_{W}$, favouring larger values of $m_{H}$. However, the overall quality of the global electroweak fit is good: $\chi^{2}=15.8$ for 13 degrees of freedom, corresponding to an overall probability of $26 \%$, and yields a $95 \%$ upper limit $m_{H}<260 \mathrm{GeV}^{39}$.

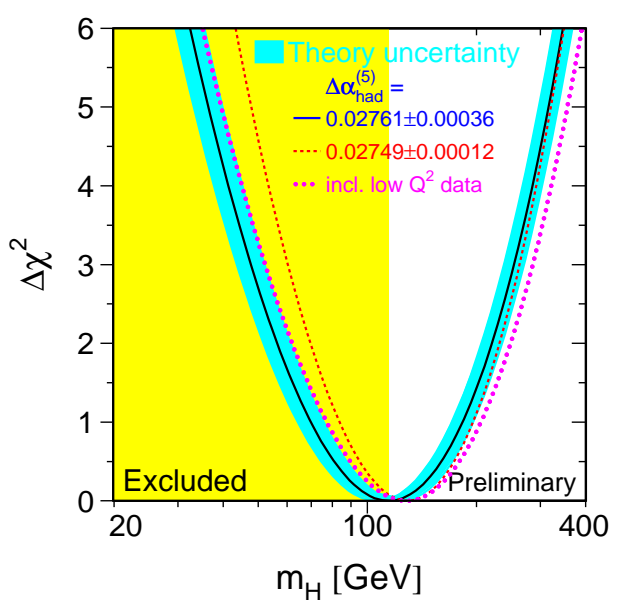

Figure 4. The $\chi^{2}$ function in the latest global fit to $m_{H} 39,44$, incorporating the new $\mathrm{D} \varnothing$ value of $m_{t}{ }^{43,42}$, the latest theoretical calculations ${ }^{45}$ and their uncertainties (blue band). Also shown are the effects of incorporating low-energy data or a theorydriven value for $\alpha_{e m}\left(m_{Z}\right)$, and the lower limit from the direct search at LEP (yellow shading) ${ }^{47}$.

The central fit value of $m_{H}$ now coincides with the lower limit from direct searches at LEP ${ }^{47}$, and the hunt for the Higgs at hadron colliders is very much on. The latest CDF upper limits on Higgs production are almost within an order of magnitude of the cross section expected in the Standard Model for $m_{H}$ between 110 and $180 \mathrm{GeV}^{43}$, and the Tevatron collider has a window of opportunity before the LHC experiments start taking data. Once they start, even a small amount of running might suffice to discover the Higgs bo- son, once the detectors are understood ${ }^{48}$.

\section{Quark Flavour Physics}

Before this conference, some of the big questions in quark flavour physics were: Are the data on quark mixing well described by the Cabibbo-Kobayashi-Maskawa (CKM) model? Are there signatures of physics beyond the Standard Model? If not, why is new physics flavour-blind? There are also some questions about the relation to neutrino flavour physics, namely why is neutrino mixing so different from quark mixing, and is there some way they might be related?

The answer to the first quark flavour question seems still to be 'yes'. The niggling $2-\sigma$ discrepancy with CKM unitarity has evaporated with a slew of new measurements of $V_{u s}$ in $K$ decays that all give values higher than the earlier PDG value ${ }^{49}$ : there is no longer any deficit of quark weak charge relative to muon decay. The previous CKM unitarity 'crisis' has disappeared ${ }^{49}$.

CKM has won again with the determination of $\sin 2 \beta$ in $b \rightarrow \bar{c} c s$ decays such as $B \rightarrow J / \psi K_{s}$ : the world average is now

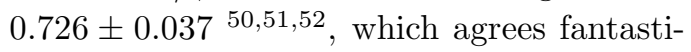
cally well with the prediction ${ }^{53,54}$ based on measurements of $K$ decay and CP-conserving $B$ properties (and relying on lattice estimates of weak matrix elements ${ }^{57}$ ), confirming that $\mathrm{CP}$ violation is 'large'. However, this success with $\sin 2 \beta$ is only the beginning of a paradigm change. CKM CP violation certainly present and large: the big issue is now to look for corrections to CKM, rather than alternatives ${ }^{54}$. Further detailed tests of CKM in clean processes are essential.

An important set of checks of the CKM model are provided by comparing the value of $\sin 2 \beta$ extracted from $b \rightarrow \bar{c} c s$ decays with the $\mathrm{CP}$-violating asymmetries found in processes dominated by $s$-penguin diagrams, including $B \rightarrow \phi K^{0}, \eta^{\prime} K_{s}, K^{+} K^{-} K_{s}, \pi^{0} K_{s}, f^{0} K_{s}$ and $\omega K_{s} 58,52,51,53,54$. These measurements are 
qualitatively consistent with $\sin 2 \beta[c c]$, in the sense that they exhibit large asymmetries of the same sign, but there are some discrepancies in the details. Thanks to new data from Belle ${ }^{52}$, in particular, $B \rightarrow \phi K_{S}$ has jumped towards the Standard Model, but $B \rightarrow \eta^{\prime} K_{s}$ is still $2.6 \sigma$ away from from $\sin 2 \beta[c c]$. Overall, the average of the $s$-penguin processes now lies $3.5 \sigma$ away from $\sin 2 \beta[c c]$. However, none of the $s$-penguin processes is as clean as $B \rightarrow J / \psi K_{s}$, and one expects departures from $\sin 2 \beta[c c]$ that are $O(15) \%$ for many modes ${ }^{54}$. Moreover, there is no hint of any direct $\mathrm{CP}$ violation in any of these modes. It would be premature to consider the $s$-penguin decays to be a 'smoking gun' for new physics.

An important step forward in checking the CKM model has been achieved at this conference with the measurement of $\alpha$ using $B \rightarrow \pi \pi^{59}, \rho \pi^{60}$ and $\rho \rho^{61}$ decays ${ }^{51,52}$, as shown in Fig. 5. The most important contributors are $B \rightarrow \rho^{+} \rho^{-51}$ (using the facts that longitudinal $\rho$ polarization states dominate and that $B \rightarrow \rho^{0} \rho^{0}$ decay is suppressed, implying that penguin pollution is small) and the Dalitz plot in $B \rightarrow \rho \pi$ decays. The world average is now $\alpha=100_{-10}^{+12}$ degrees, which is consistent with, and more accurate than, the CKM prediction $\alpha=98 \pm 16$ degrees. However, the direct determinations $\gamma=77_{-19}^{+17} \pm$ $13 \pm 11$ (Belle), $88 \pm 41 \pm 19 \pm 10$ (BABAR) degrees ${ }^{62}$ are still some way from challenging the CKM model.

Another important result reported here by Belle and BABAR has been the observation of direct $\mathrm{CP}$ violation in $B^{0} \rightarrow K^{+} \pi^{-}$ and conjugate decays: $A_{C P}\left(K^{+} \pi^{-}\right)=$ $0.114 \pm 0.020$, a $4.2-\sigma$ effect $63,52,51$. This is only the second observation of direct $\mathrm{CP}$ violation, following that in $K^{0} \rightarrow \pi \pi$ decays ${ }^{49}$, and is the first in $B$ decays. It confirms that these cannot be described by a superweak theory, and is consistent with the CKM model. However, this measurement poses a problem for the factorization scheme ${ }^{64}$, and

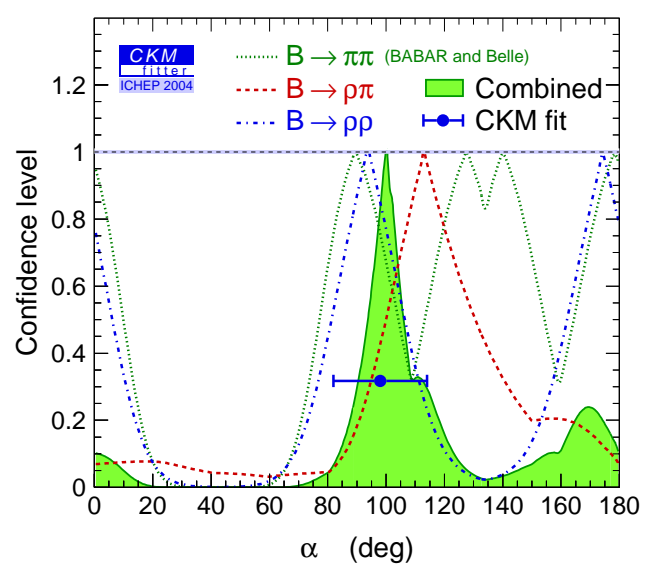

Figure 5. Prediction for the angle $\alpha$ from a global CKM fit ${ }^{54,56}$, compared with recent experimental determinations $51,52,59,60,61$.

is somewhat different from the asymmetry in $B^{+}$decays: $A_{C P}\left(K^{+} \pi^{-}\right)=0.04 \pm 0.05 \pm 0.02$. This difference might be laid at the door of some coalition of electroweak penguins, nonperturbative effects and final-state interactions ${ }^{54}$.

Putting together all the available information on quark flavour physics, there is excellent consistency with the CKM model, as seen in Fig. 6. Overall, there is little room left for new physics ${ }^{54}$, as seen in Fig. 7. Whatever there is, it probably has a similar flavour structure similar to that of CKM, which is a potential challenge for extensions of the Standard Model such as supersymmetry ${ }^{65}$.

However, there is still significant room for future progress, since the experimental errors in many $B$ decay modes are still considerably larger than the theoretical uncertainties ${ }^{54}$. Any larger discrepancy would be evidence for new physics. Theoretical breakthroughs would be needed to push the present theoretical errors much lower, but these decay modes will not be theory-limited for a long time.

Before leaving quark flavour physics, it should be mentioned that important consistency checks on the CKM model may be made in charm physics ${ }^{66}$ and using rare $K$ 


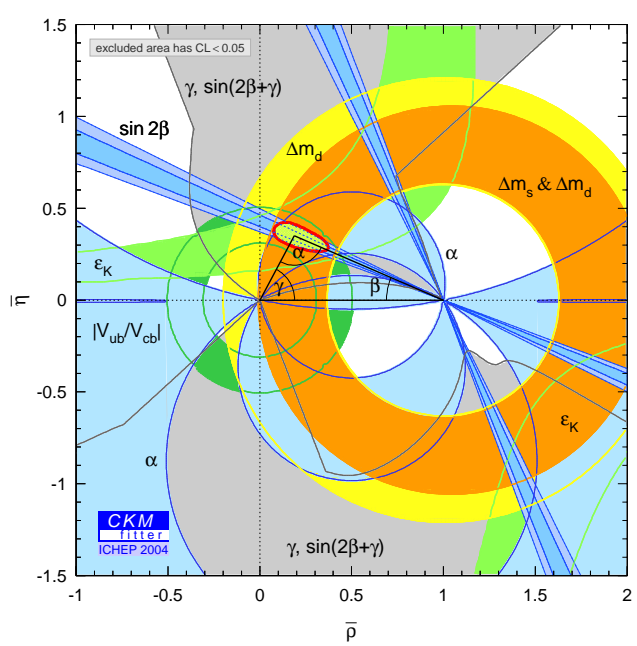

Figure 6. Measurements of $\mathrm{CP}$ violation in $B$ decays ${ }^{51,52}$ are in excellent agreement with the predictions of a global CKM fit ${ }^{54,56}$.

decays, particularly $K \rightarrow \pi \nu \bar{\nu}$ decays (in both charged and neutral modes) ${ }^{49}$. Three candidate $K^{+} \rightarrow \pi^{+} \nu \bar{\nu}$ decays have now been seen ${ }^{67}$, and the rate has been sinking towards the SM value, while progress is expected soon in the search for $K_{L} \rightarrow \pi^{0} \nu \bar{\nu}$ decay. The related $K_{L} \rightarrow \pi \ell^{+} \ell^{-}$decays are also interesting for studies of $\mathrm{CP}$ violation, and important groundwork for their interpretation is being played by NA48 measurements of $K_{S} \rightarrow \pi^{0} \ell^{+} \ell^{-}$and $\pi^{0} \gamma \gamma{ }^{68}$. These constrain amplitudes that compete with the direct $\mathrm{CP}$-violating amplitude expected in the CKM model.

\section{Neutrino Masses and Oscillations}

Neutrinos provided the first confirmed physics beyond the Standard Model 1,69, namely confirmed deficits in both the atmospheric and solar neutrino fluxes. Another apparent deficit has been observed by the LSND experiment at Los Alamos ${ }^{70}$, for which we are awaiting confirmation from the MiniBooNE experiment at Fermilab ${ }^{71}$.

One of the most exciting developments

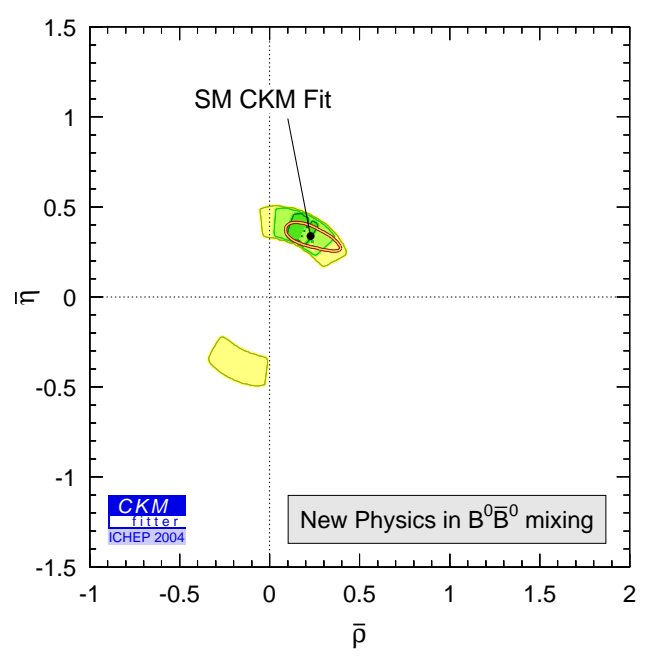

Figure 7. The agreement between $\mathrm{CP}$ violation measurements ${ }^{51,52}$ and the global CKM fit ${ }^{56}$ leaves little room for new physics 54 .

in neutrino physics reported here ${ }^{72}$ has been the evidence for neutrino oscillation patterns found by both the Super-Kamiokande ${ }^{73}$ and KamLAND ${ }^{74}$ experiments, shown in Fig. 8. Super-Kamiokande made a specific study of atmospheric neutrino events with a good determination of $L / E$, and observed an apparent oscillation dip. Rival hypotheses such as neutrino decay and decoherence are disfavoured by $\chi^{2}$ (neutrino decay - oscillation) $=11.4$ and $\chi^{2}$ (neutrino decoherence - oscillation $)=14.6^{73}$. Evidence of similar strength has been reported by the KamLAND experiment ${ }^{74}$ and there is also a hint of an oscillation dip in $\mathrm{K} 2 \mathrm{~K}$ data ${ }^{75}$. Rivals to the oscillation hypothesis are falling by the wayside.

Within the oscillation framework, the new, higher-statistics KamLAND data are strikingly consistent with the Super-

Kamiokande and SNO data on solar neutri$\operatorname{nos}^{72}$, with a similar mass-squared difference $\Delta m^{2} \sim 8 \times 10^{-5} \mathrm{eV}^{2}$ and compatible mixing angles. Likewise, accelerator neutrino data from K2K ${ }^{76}$ confirm Super-Kamiokande on atmospheric neutrinos, with $\Delta m^{2} \sim 2.5 \times$ $10^{-3} \mathrm{eV}^{2}$, and are also compatible with max- 

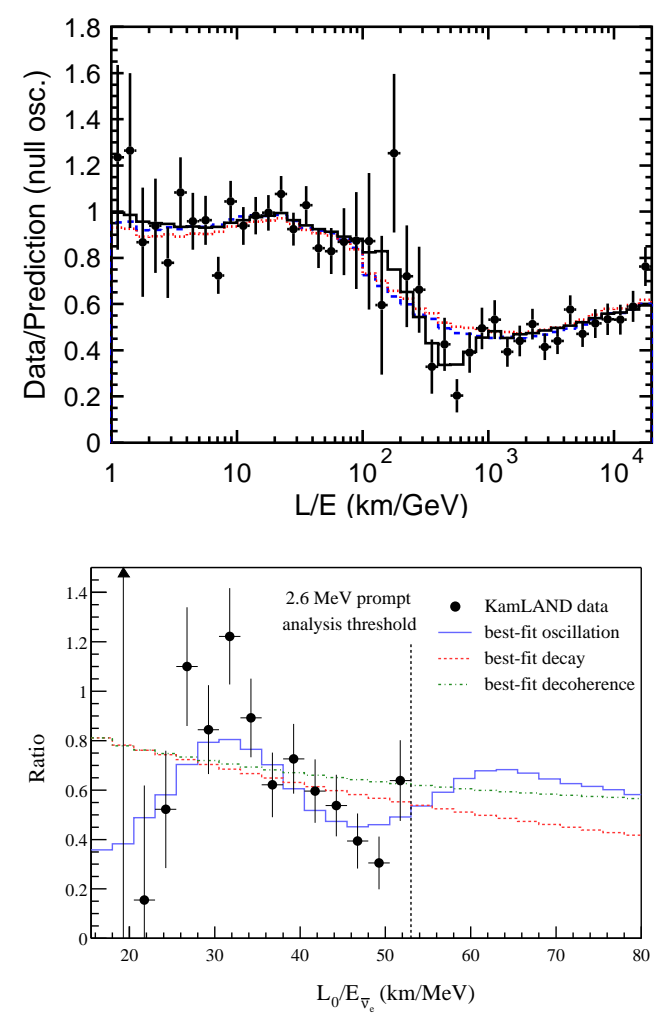

Figure 8. Chracteristic oscillatory patterns have been seen in atmospheric neutrino data (upper panel) ${ }^{73}$ and in reactor neutrino data (lower panel) ${ }^{74}$.

imal mixing.

Interpreted in terms of neutrino oscillations, the atmospheric and solar neutrino data indicate a (near) bimaximal mixing pattern completely different from that found for quarks ${ }^{69}$. Empirically, the data are compatible with the relation $\Theta_{\odot}+\Theta_{c}=\pi / 4$, and a challenge for theorists is to figure out why this should be the case ${ }^{77}$. In addition to the two large mixing angles already observed, there is a third mixing angle, $\Theta_{13}$, whose measurement remains a challenge for future accelerator or reactor experiments ${ }^{76}$. The Holy Grail in neutrino oscillations is the search for CP violation, but we now realize that this may have only an indirect relation to cosmology via baryogenesis ${ }^{78}$.

Much still remains to do! Oscillations are made possible by differences in neutrino masses, and we want to know whether they follow a normal or inverted hierarchy. At a basic level, theorists speculate whether neutrino masses are of Dirac or Majorana type. Many models are based on the seesaw mechanism, but there is not a shred of evidence for it! Insight into these questions can be provided indirectly by searches for neutrinoless double- $\beta$ decay and searches for the violation of charged lepton numbers ${ }^{69}$.

\section{Physics beyond the Standard Model}

The most pressing issue in physics beyond the Standard Model is breaking electroweak symmetry (EWSB) ${ }^{1}$. This problem must be solved below $\sim 1 \mathrm{TeV}$, and its solution would be a revolution in fundamental physics that is a necessary basis for further theoretical speculations. There are various hints of grand unification, such as the possible unification of gauge couplings and also neutrino masses, but it is difficult to test such unification ideas directly, and here I follow the rapporteur ${ }^{1}$ in focussing on EWSB.

If we adopt the calculability principle that the electroweak scale should be calculable in terms of other physical mass scales, we are led to consider models without quadratic divergences in the mass of the Higgs boson. Then the two basic options are to invoke supersymmetry or to interpret the Higgs boson as a pseudo-Goldstone boson ${ }^{1}$. Supersymmetry has the supplementary advantages that it facilitates unification of the gauge couplings ${ }^{79}$ and supplies a natural candidate for astrophysical dark matter ${ }^{80}$. LEP data require some fine-tuning of the supersymmetric model parameters, but (in contrast to some others) I do not regard this as a severe problem ${ }^{81}$.

What are the alternatives to the default option of a light Higgs boson accompanied by supersymmetry? One possibility considered has been to re-examine the naïve interpre- 
tation of the electroweak data ${ }^{83}$. Questions have been raised about the consistency of the precision electroweak measurements, and it has been asked whether some should be discarded, though, as already mentioned, the 'discrepancy' between the direct and indirect limits on $m_{H}$ have now evaporated ${ }^{39,44}$. As already remarked, heavy-flavour measurements alone tend to favour a heavier Higgs boson than is allowed by simple supersymmetric models. Another possibility is that the global electroweak fit should include contributions from higher-dimensional operators ${ }^{82}$ as well as the Higgs boson. Analyses of this type find allowed corridors of parameter space extending to higher Higgs masses. If the Higgs boson is indeed light, alternatives to supersymmetry are provided by little Higgs models ${ }^{84}$, in which the cancellation of one-loop quadratic divergences is ensured by an extra 'top-like' quark, more gauge bosons, and Higgs-like fields. At the other extreme, theorists have been experimenting with Higgsless models ${ }^{85}$, in which WW scattering becomes strong at high energies. Such models have problems with the precision electroweak data, which may be alleviated in models formulated with extra dimensions, though problems still remain.

Among these various options, little Higgs models may be the most interesting, so I discuss them in a bit more detail ${ }^{84}$. The Standard Model is embedded in a larger gauge group that is broken down to $S U(2) \times U(1)$, with the light Higgs boson appearing as a pseudo-Goldstone boson. The quadratic correction due to the top quark:

$$
\delta m_{H, t o p}^{2}(S M) \sim(115 \mathrm{GeV})^{2}\left(\frac{\Lambda}{400 \mathrm{GeV}}\right)^{2}
$$

is cancelled, as seen in Fig. 9, by a new heavy $T$ quark with mass

$$
m_{T}>2 \lambda_{t} f \sim 2 f
$$

where $f>1 \mathrm{TeV}$. As a result, the above quadratic divergence is softened to a logarith- mic one:

$$
\begin{aligned}
\delta m_{H, t o p}^{2}(L H) & \sim \frac{6 G_{F} m_{t}^{2}}{\sqrt{2} \pi^{2}} m_{T}^{2} \log \frac{\Lambda}{m_{T}} \\
& \geq 1.2 f^{2}
\end{aligned}
$$

There is an upper bound on the mass of the new $T$ quark:

$$
M_{T}<2 \mathrm{TeV}\left(m_{H} / 200 \mathrm{GeV}\right)^{2}
$$

There are also upper bounds on the expected new gauge bosons and Higgs bosons:

$$
\begin{aligned}
M_{W^{\prime}} & <6 \mathrm{TeV}\left(m_{H} / 200 \mathrm{GeV}\right)^{2}, \\
M_{H^{++}} & <10 \mathrm{TeV} .
\end{aligned}
$$

More physics must appear above the $10 \mathrm{TeV}$ scale, so such a little Higgs theory is not as complete as supersymmetry.
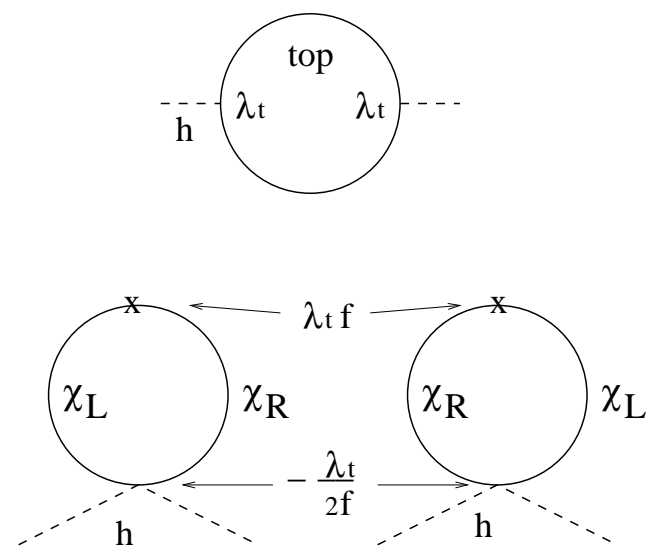

Figure 9. In 'Little Higgs' models, the quadraticallydivergent top-quark loop contribution to $m_{H}^{2}$ is cancelled by loops containing a heavier charge- $2 / 3$ quark ${ }^{84}$.

Is there any evidence from accelerator experiments for physics beyond the Standard Model? The best claimant is the measurement of $g_{\mu}-2$ at BNL ${ }^{86}$, but its interpretation has been clouded ${ }^{39}$ by uncertainties in the Standard Model contribution calculated on the basis of the hadronic vacuum polarization extracted from $e^{+} e^{-}$data or $\tau$ decay data ${ }^{87}$. The largest contributions to $g_{\mu}-2$ and the most important uncertainties 
come from low energies $<1 \mathrm{GeV}$. In an important recent development, KLOE has released data ${ }^{88}$ that agree well with those from CMD-2 ${ }^{89}$, and the trend is now to discard the $\tau$ data in the Standard Model evaluation of $g_{\mu}-2^{39}$, although this begs the question why there is a $\sim 10 \%$ discrepancy between the $\tau$ and $e^{+} e^{-}$data between the $\rho$ peak and $1 \mathrm{GeV}$. However, using the final BNL data and dropping the $\tau$ data, one now finds a discrepancy with the Standard Model of 39

$$
a_{\mu}^{e x p}-a_{\mu}^{S M}=(25.2 \pm 9.2) \times 10^{-10},
$$

corresponding to a $2.7 \sigma$ effect. This is promising, but insufficient to make a convincing claim for physics beyond the Standard Model. Nevertheless, it provides a significant constraint on many extensions of the Standard Model, such as supersymmetry.

Many direct searches for physics beyond the Standard Model were also reported here, and I mention in particular the renewed search for squarks and gluinos at the Tevatron collider using Run 2 data ${ }^{90}$. General searches have now edged beyond the Run 1 limits, to 292 and $333 \mathrm{GeV}$ for squarks and gluinos in one analysis, and a dedicated search for a light sbottom squark excludes a significant new region of parameter space ${ }^{90}$. Further progress from Run 2 is eagerly awaited.

We did not have a plenary talk about physics prospects for the LHC, but some were presented in a parallel session ${ }^{48}$. Following discovery (!?), several sparticle masses may be measured quite accurately at the LHC in exclusive cascade decays ${ }^{91}$. Measurements of mass differences will typically be limited by the detector performance to errors of order $1 \%$, whereas the error in the overall mass scale will be dominated by that in the unknown missing energy to the order of $10 \%{ }^{48}$. New simulations indicate that it should be possible to measure sparticle spins at the LHC, in some cases. Analayses have shown how to measure the spin- $1 / 2$ nature of the $\chi_{2}$, and it has been shown that one could also measure the scalar nature of the slepton at several distinct points in parameter space ${ }^{48}$.

However, digging a supersymmetric signal out from the Standard Model backgrounds will not be easy, and it is important to calculate the latter as accurately as possible. There are plenty of necessary but unglamorous NLO QCD calculations ${ }^{2}$ waiting to be done! Perhaps the new string techniques will simplify them?

As we heard from the rapporteur ${ }^{1}$, the LHC has an important location on the roadmap for exploring EWSB physics, and there are many scenarios in which a $500-\mathrm{GeV}$ LC could add significant physics value. If naturalness is a good guide, the LHC will find signals of new physics. For their full interpretation, a 500-GeV LC would often (though not always) be very significant.

\section{A Few Remarks on String Theory}

String theory is not only very beautiful mathematics, but also provides very powerful tools for field-theoretical calculations, e.g., for QCD as discussed earlier ${ }^{2}$. String theory has also aready solved many major problems in Quantum Gravity, such as the extraction of sensible results from perturbative calculations and the counting of black hole states ${ }^{92}$. String theory might explain the origin of the Universe. It might replace our naive models of inflation based on an elementary scalar field ${ }^{93}$. However, our concern here is whether it is relevant to particle physics. This is an open question, but there are many exciting possibilities. The landscape of string vacua is very rich ${ }^{94}$, and one or more of these states may be able to describe all of particle physics. However, we are still lacking a distinctive experimental signature. If they are ever discovered, extra dimensions might provide such a 'smoking gun', but this remains to be seen. 


\section{Particle Astrophysics and Cosmology}

Cosmology and high-energy astrophysics abound in important problems ${ }^{95}$ that only particle physics may be able to solve: What is the Dark Matter? Is there Dark Energy? Are there ultra-high-energy cosmic rays (UHECRs) beyond the GZK cutoff? Was there inflation? How did the Universe begin?

According to the concordance model of cosmology, about $73 \%$ of the energy density of the Universe is unclumped, invisible Dark Energy, about $23 \%$ is clumped, invisible Dark Matter, and only about $4 \%$ is visible matter, with neutrinos an even smaller percentage ${ }^{95}$. This concordance model has been supported by data on large-scale structures ${ }^{96}$, highredshift supernovae ${ }^{97}$ and observations of the cosmic microwave background by WMAP et al. ${ }^{98}$. As we heard at this conference ${ }^{99}$, gamma-ray bursters are now emerging as another standard, normalizable candle capable of measuring the geometry of the Universe ${ }^{100}$, and they tend also to support this concordance model.

There has been continuing discussion here of particle candidates for the cold Dark Matter ${ }^{95}$. These include the axion, WIMPs such as the lightest supersymmetric particle (LSP) - which might be either a neutralino or a gravitino, the lightest Kaluza-Klein Particle (LKP) in models with universal extra dimensions, and a superheavy (metastable) 'WIMPzilla' particle, which might have been produced at inflation, and whose decays could be responsible for the UHECRs. Here I focus on the neutralino WIMP possibility.

There has been significant recent progress in the direct search for WIMP Dark Matter, looking for elastic scattering on nuclei in low-background experiments. The previous DAMA modulation signal is increasingly difficult to reconcile with other experiments such as CDMS2 ${ }^{101}$, which currently has the best upper limit on Dark Matter scat- tering, as shown in Fig. 10. This experiment is already starting to reach into the range expected in realistic models, and has good prospects for further improvement by a factor $\sim 20$.

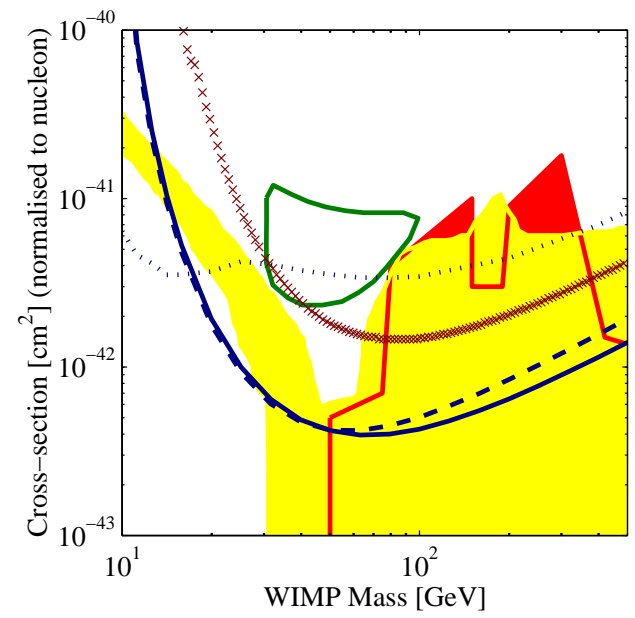

Figure 10. Upper limits on the cross section for spinindependent scattering between Dark Matter particles and nucleons compared with some theoretical models 101 .

There have been discussions here of a possible signal of gamma rays coming from neutralino annihilations near the galactic centre, since EGRET has observed an apparent excess over standard cosmic-ray calculations ${ }^{102,103}$. However, there are large uncertainties in the cosmic-ray background calculated with the standard Galprop code, and there are also large unknowns in the prospective signal normalization, so my advice is to 'wait and see'.

A new upper limit was reported here by AMANDA on muons produced by highenergy neutrinos emanating from neutralino annihilations inside the Sun ${ }^{104}$, which is comparable with previous limits from BAKSAN, MACRO and Super-Kamiokande. The IceCube experiment currently under construction has good prospects to explore far into the range expected in realistic models.

There are prospects that one of these Dark Matter experiments might be able to 
rival the LHC in the search for supersymmetry ${ }^{105}$.

\section{Future Accelerators}

There are many complex issues related to future accelerators, many of which are not technical. In some ways, the simplest question is what accelerators we want to do the physics ${ }^{106}$. It seems that we know enough to build interesting accelerators 107 and to do experiments with the accelerators currently being proposed ${ }^{108}$, but it is less obvious that they will be able to answer all our questions ${ }^{1}$. But we also have to figure out how we can continue to involve a diversity of regions, and how we can ensure a diversity of facilities. Certainly we shall need to work together to get them approved, and also to build them ${ }^{109}$. Finally, I should like to add another question: How can we ensure access to new accelerators from all qualified and interested physicists? We have recently had instances of scientists from some countries being denied visas to attend a major international conference, and IUPAP has announced that it will not sponsor conferences where open attendance cannot be assured ${ }^{110}$. What would be the attitude of IUPAP to the construction of a unique global accelerator in a country that is not open to scientists from around the world?

There are plenty of strong motivations for future colliders, since both physics and cosmology lead us to expect strongly new physics at the scale of $1 \mathrm{TeV}^{106}$. As we have heard from the rapporteur here, the physics case for the LHC has been made and accepted, and it will look into the whole region where new physics can be expected. The physics case for a $\mathrm{TeV}$ International Linear Collider (ILC) has also been made, and the physics cases for CLIC (and perhaps a larger hadron collider) will be understood better following the results from the LHC (and perhaps a TeV ILC) ${ }^{106}$.
The principal tasks for a TeV ILC are well understood ${ }^{106}$. It should measure $m_{t}$ with an accuracy $<100 \mathrm{MeV}$. If there is a light Higgs boson of any kind, the ILC should pin it down: What is its precise mass and does it have Standard Model couplings? As seen in Fig. 11, the decays of a light Higgs - if it exists - could be measured very accurately at the ILC. Moreover, if there are extra light particles, such as those predicted by supersymmetry, the ILC would also measure their masses and properties very well. On the other hand, if the LHC sees nothing new below $\sim 500 \mathrm{GeV}$, the ILC should look for indirect signatures, such as those of a new $Z^{\prime}$ or a high-mass $W_{L} W_{L}$ resonance.

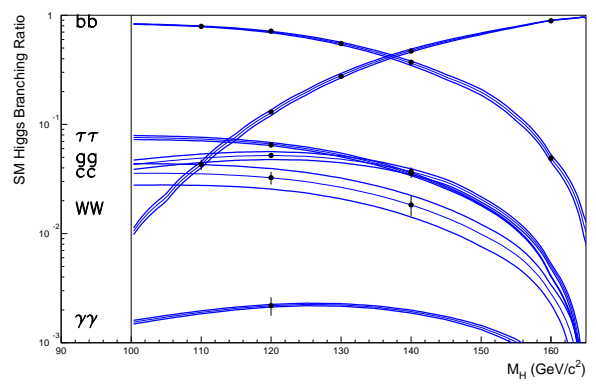

Figure 11. The decay branching ratios of a light Higgs boson could be measured very accurately with the ILC ${ }^{106}$.

One of the most dramatic developments here has been the announcement ${ }^{109}$ of the ITRP recommendation that the ILC be based on superconducting $r f$ technology ${ }^{111}$, and its endorsement by ICFA. We have been told that the features of the superconducting technology that tipped the balance in its favour follow in part from the low $r f$ frequency. The hope is now that the final design of the ILC will be developed by a team drawn from the combined warm and cold linear collider communities, taking full advantage of the experience and expertise of both ${ }^{107}$.

Beyond the sub-TeV ILC, we believe we shall need a multi-TeV linear collider ${ }^{112}$, for which the only current contender is CLIC 
with a nominal design energy of $3 \mathrm{TeV} 106$. CLIC is based on a novel two-beam accelerator concept, rather than conventional klystrons. Two CLIC test facilities have already operated successfully, and third CLIC test facility has now entered operation. It is planned to pass all the showstopping R1 and R2 R\& D milestones, by 2009 if sufficient resources can be found, about five years after the ILC.

Although the physics agenda for CLIC cannot yet be completely defined, I am convinced that it will be very rich. Consider just the example of supersymmetry. The threshold for supersymmetry may be within the reach of the sub-TeV ILC, but, as seen in Fig. 12, this cannot be guaranteed ${ }^{113}$. Even if some supersymmetric particles do have masses low enough to be observed at the ILC, most of the spectrum will lie beyond its reach, and CLIC would be the natural machine to study it ${ }^{114,115}$.

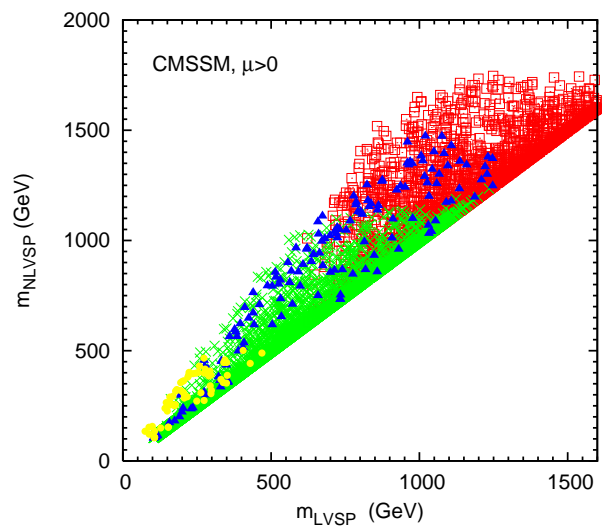

Figure 12. Scatter plot of the masses of the lightest visible supersymmetric particle (LVSP) and next-tolightest visible supersymmetric particle (NVSP) in the constrained MSSM with universal input scalar masses ${ }^{113}$ : similar results were found in other supersymmetric scenarios. The blue points have an appropriate Dark Matter density, the green points should be accessible to the LHC, and the yellow points might be detectable directly in Dark Matter scattering experiments.

\section{Summary of the Summary}

As we have heard at this conference, QCD is becoming ever more quantitative, but there remain qualitative puzzles, particularly in spectroscopy. Electroweak theory and experiment suggest new physics at the TeV scale, very likely a Higgs boson and perhaps many other particles in addition. Flavour physics is also becoming more quantitative, and the highlight of this conference has been that the CKM is looking better and better all the time. We have also seen here that neutrinos really do oscillate. The symbiosis with cosmology is growing, and the LHC is on its way. There are many good ideas for future accelerators, and the ITRP has done its work in choosing a technology for the ILC. Now is the time for us to get back to our own work!

\section{References}

1. R. Barbieri, plenary talk at this conference.

2. W. J. Stirling, plenary talk at this conference.

3. D. Lucchesi, plenary talk at this conference.

4. M. Klein, plenary talk at this conference.

5. M. Cacciari, S. Frixione, M. L. Mangano, P. Nason and G. Ridolfi, JHEP 0407 (2004) 033 [arXiv:hep-ph/0312132].

6. S. Bethke, arXiv:hep-ex/0407021.

7. A. Vogt, S. Moch and J. Vermaseren, Nucl. Phys. B 691 (2004) 129 [arXiv:hep-ph/0404111]; arXiv:hep-ph/0407321.

8. F. Cachazo, P. Svrcek and E. Witten, JHEP 0409 (2004) 006 [arXiv:hepth/0403047]; arXiv:hep-th/0406177; arXiv:hep-th/0409245; G. Georgiou, E. W. N. Glover and V. V. Khoze, JHEP 0407 (2004) 048 [arXiv:hepth/0407027].

9. S. J. Parke and T. R. Taylor, Phys. Rev. Lett. 56 (1986) 2459. 
10. For a review, see V. V. Khoze, arXiv:hep-th/0408233.

11. J. Dunlop, plenary talk at this conference; T. Hallman and C. M. Ko, talks in the 'Heavy Ions' parallel session at this conference.

12. Several relevant theoretical papers will appear in the proceedings of the Workshop on New Discoveries at RHIC: The Current Case for the Strongly Interactive QGP, Brookhaven, Upton, New York, 14-15 May 2004, see, for example: J. P. Blaizot and F. Gelis, arXiv:hep-ph/0405305; M. Gyulassy and L. McLerran, arXiv:nucl-th/0405013; E. V. Shuryak, quark-gluon arXiv:hepph/0405066; H. Stoecker, arXiv:nuclth/0406018; X. N. Wang, arXiv:nuclth/0405017.

13. J. Adams et al. [STAR Collaboration], arXiv:nucl-ex/0407007.

14. S. Jin, plenary talk at this conference.

15. For me, one of the most appealing approaches is based on chiral symmetry: W. A. Bardeen, E. J. Eichten and C. T. Hill, Phys. Rev. D 68 (2003) 054024 [arXiv:hep-ph/0305049].

16. A. V. Evdokimov et al. [SELEX Collaboration], arXiv:hep-ex/0406045; confirmation of this state is eagerly awaited.

17. A. Airapetian et al. [HERMES Collaboration], Phys. Lett. B 585 (2004) 213 [arXiv:hep-ex/0312044].

18. S. Chekanov et al. [ZEUS Collaboration], Phys. Lett. B 591 (2004) 7 [arXiv:hep-ex/0403051].

19. A. Aktas et al. [H1 Collaboration], Phys. Lett. B 588 (2004) 17 [arXiv:hepex/0403017]; confirmation of this state is eagerly awaited.

20. C. Alt et al. [NA49 Collaboration], Phys. Rev. Lett. 92 (2004) 042003 [arXiv:hep-ex/0310014]; confirmation of this state is eagerly awaited.

21. F. E. Close, plenary talk at this conference. See also, H. Lipkin, talk in the
'Hadron Spectroscopy' parallel session at this conference.

22. Q. Zhao and F. E. Close, arXiv:hep$\mathrm{ph} / 0404075$.

23. R. N. Cahn and G. H. Trilling, Phys. Rev. D 69 (2004) 011501 [arXiv:hep$\mathrm{ph} / 0311245]$.

24. B. Aubert et al. [BABAR Collaboration], arXiv:hep-ex/0408064; V. Halyo, talk in the 'Hadron Spectroscopy' parallel session at this conference.

25. However, M. Danilov, talk in the 'Hadron Spectroscopy' parallel session at this conference, reported negative results from a low-energy search in secondary interactions produced inside the Belle detector.

26. See also H. J. Lipkin, arXiv:hep$\mathrm{ph} / 0409349$.

27. R. L. Jaffe and F. Wilczek, Phys. Rev. Lett. 91 (2003) 232003 [arXiv:hep$\mathrm{ph} / 0307341]$.

28. M. Karliner and H. J. Lipkin, Phys. Lett. B 575 (2003) 249 [arXiv:hep$\mathrm{ph} / 0402260]$.

29. D. Diakonov, V. Petrov and M. V. Polyakov, Z. Phys. A 359 (1997) 305 [arXiv:hep-ph/9703373].

30. T. H. R. Skyrme, Proc. Roy. Soc. Lond. A 260 (1961) 127 and Nucl. Phys. 31 (1962) 556.

31. B. Q. Ma, talk in the 'Hadron Spectroscopy' parallel session at this conference; see also J. R. Ellis, M. Karliner and M. Praszalowicz, JHEP 0405 (2004) 002 [arXiv:hep-ph/0401127].

32. S. K. Choi et al. [Belle Collaboration], Phys. Rev. Lett. 91 (2003) 262001 [arXiv:hep-ex/0309032]; K. Abe et al. [Belle Collaboration], arXiv:hepex/0408116.

33. D. Acosta et al. [CDF II Collaboration], Phys. Rev. Lett. 93 (2004) 072001 [arXiv:hep-ex/0312021].

34. B. Aubert et al. [BABAR Collaboration], arXiv:hep-ex/0406022. 
35. K. Abe et al. [Belle Collaboration], arXiv:hep-ex/0408126.

36. J. Z. Bai et al. [BES Collaboration], Phys. Rev. Lett. 91 (2003) 022001 [arXiv:hep-ex/0303006], arXiv:hep-ex/0304001; M. Ablikim et al. [BES Collaboration], arXiv:hepex/0405050.

37. The BES report of a low-mass $\sigma$ resonance in the $\pi \pi$ system: M. Ablikim et al. [BES Collaboration], arXiv:hep-ex/0406038 is quite consistent with a previous experimental report: E. M. Aitala et al. [E791 Collaboration], Phys. Rev. Lett. 86 (2001) 770 [arXiv:hep-ex/0007028]; and with theoretical calculations based on chiral symmetry: G. Colangelo, J. Gasser and H. Leutwyler, Nucl. Phys. B 603 (2001) 125 [arXiv:hep-ph/0103088].

38. K. Abe et al. [BELLE Collaboration], three-body arXiv:hep-ex/0409009 and arXiv:hep-ex/0409010.

39. F. Teubert, plenary talk at this conference.

40. G. P. Zeller et al. $[\mathrm{NuTeV}$ Collaboration], Phys. Rev. Lett. 88 (2002) 091802 [Erratum-ibid. 90 (2003) 239902] [arXiv:hep-ex/0110059].

41. NOMAD Collaboration, http://nomad-info.web.cern.ch/ nomad-info/.

42. V. M. Abazov et al. [D0 Collaboration], Nature 429 (2004) 638 [arXiv:hepex/0406031].

43. D. Denisov, plenary talk at this conference.

44. V. M. Abazov et al. [D0 Collaboration], Nature 429 (2004) 638 [arXiv:hepex/0406031].

45. A. Djouadi and C. Verzegnassi, Phys. Lett. B 195 (1987) 265; A. Djouadi, Nuovo Cim. $\quad$ A 100 (1988) 357; B. Kniehl, Nucl. Phys. B 347 (1990) 89; F. Halzen and B. Kniehl, Nucl. Phys. B 353 (1991) 567; B. Kniehl and
A. Sirlin, Nucl. Phys. B 371 (1992) 141; Phys. Rev. D 47 (1993) 883; K. Chetyrkin, J.H. Kühn and M. Steinhauser, Phys. Rev. Lett. $\mathbf{7 5}$ (1995) 3394, hep-ph/9504413; L. Avdeev et al., Phys. Lett. B 336 (1994) 560, hepph/9406363; E: Phys. Lett. B 349 (1995) 597. K. Chetyrkin, J.H. Kühn and M. Steinhauser, Nucl. Phys. B 482 (1996) 213, hep-ph/9606230.

46. J. Z. Bai et al. [BES Collaboration], Phys. Rev. Lett. 88 (2002) 101802 [arXiv:hep-ex/0102003].

47. R. Barate et al. [ALEPH Collaboration, DELPHI Collaboration, L3 Collaboration, OPAL Collaboration and LEP Working Group for Higgs boson searches], Phys. Lett. B 565 (2003) 61 [arXiv:hep-ex/0306033].

48. A. J. Barr, talk in the 'Beyond the Standard Model' parallel session at this conference.

49. V. Patera, plenary talk at this conference. See also C. Scarlett, parallel session talk at this conference.

50. K. Abe et al. [BELLE Collaboration], arXiv:hep-ex/0408111; arXiv:hepex/0408127. M. Bruinsma, T. Higuchi, talks in the 'CP Violation' parallel session at this conference.

51. M. Giorgi, plenary talk at this conference.

52. Y. Sakai, plenary talk at this conference.

53. A. Ali, plenary talk at this conference.

54. Z. Ligeti, plenary talk at this conference, arXiv:hep-ph/0408267.

55. M. Bona et al. [UTfit Collaboration], arXiv:hep-ph/0408079; M. Bona, talk in the ' $\mathrm{CP}$ Violation' parallel session at this conference.

56. J. Charles et al. [CKMfitter Group Collaboration], arXiv:hep-ph/0406184; M. Pivk, talk in the 'CP Violation' parallel session at this conference.

57. S. Hashimoto, plenary talk at this conference. 
58. B. Aubert et al. [BABAR Collaboration], arXiv:hep-ex/0408072; arXiv:hepex/0408076; arXiv:hep-ex/0408090; arXiv:hep-ex/0408095; K. Abe [the Belle Collaboration], arXiv:hep-ex/0409049; A. Höcker, talk in the ' $\mathrm{CP}$ Violation' parallel session at this conference.

59. K. Abe et al. [Belle Collaboration], Phys. Rev. Lett. 93 (2004) 021601 [arXiv:hep-ex/0401029]; B. Aubert et al. [BABAR Collaboration], arXiv:hepex/0408081; arXiv:hep-ex/0408089.

60. C. C. Wang et al. [Belle Collaboration], arXiv:hep-ex/0408003; B. Aubert et al. [BABAR Collaboration], arXiv:hepex/0408099; M. Cristinziani, A. Somov, talks in the 'CP Violation' parallel session at this conference.

61. B. Aubert et al. [BABAR Collaboration], arXiv:hep-ex/0404029; arXiv:hepex/0408061; C. Dallapiccola, talk in the 'CP Violation' parallel session at this conference.

62. G. Cavoto, A. Bozek, talks in the 'CP Violation' parallel session at this conference.

63. B. Aubert et al. [BaBar Collaboration], arXiv:hep-ex/0407057; Y. Chao et al. [Belle Collaboration], arXiv:hepex/0408100.

64. M. Beneke, G. Buchalla, M. Neubert and C. T. Sachrajda, Nucl. Phys. B 591 (2000) 313 [arXiv:hep-ph/0006124].

65. A. Masiero and O. Vives, New J. Phys. 4 (2002) 4.

66. I. Shipsey, plenary talk at this conference.

67. V. V. Anisimovsky et al. [E949 Collaboration], Phys. Rev. Lett. 93 (2004) 031801.

68. A. Lai et al. [NA48 Collaboration], decay," Phys. Lett. B 578 (2004) 276 [arXiv:hep-ex/0309022]; J. R. Batley et al. [NA48/1 Collaboration], Phys. Lett. B 576 (2003) 43 [arXiv:hep-ex/0309075] and CERN PH-EP/2004-025, to be pub- lished in Phys. Lett. B.

69. P. Langacker, plenary talk at this conference.

70. C. Athanassopoulos et al. [LSND Collaboration], Phys. Rev. Lett. 81 (1998) 1774 [arXiv:nucl-ex/9709006].

71. A. A. Aguilar-Arevalo [MiniBoonE Collaboration], arXiv:hep-ex/0408074; E. J. Prebys, talk in the 'Neutrino Physics' parallel session at this conference.

72. Y.-f. Wang, plenary talk at this conference.

73. Y. Ashie et al. [Super-Kamiokande Collaboration], arXiv:hep-ex/0404034; C. Saji, talk in the 'Neutrino Physics' parallel session at this conference.

74. T. Araki et al. [KamLAND Collaboration], arXiv:hep-ex/0406035; I. Shimizu, talk in the 'Neutrino Physics' parallel session at this conference.

75. M. H. Ahn et al. [K2K Collaboration], Phys. Rev. Lett. 90 (2003) 041801 [arXiv:hep-ex/0212007]; updated with higher statistics in the talk by $\mathrm{T}$. Ishida in the 'Neutrino Physics' parallel session at this conference.

76. C. McGrew, plenary talk at this conference.

77. W. Rodejohann, talk in the 'Neutrino Physics' parallel session at this conference.

78. See, e.g., J. R. Ellis and M. Raidal, Nucl. Phys. B 643 (2002) 229 [arXiv:hep$\mathrm{ph} / 0206174]$.

79. J. R. Ellis, S. Kelley and D. V. Nanopoulos, Phys. Lett. B 249 (1990) 441 and Phys. Lett. B 260 (1991) 131; U. Amaldi, W. de Boer and H. Furstenau, Phys. Lett. B 260 (1991) 447; P. Langacker and M. x. Luo, Phys. Rev. D 44 (1991) 817; C. Giunti, C. W. Kim and U. W. Lee, Mod. Phys. Lett. A 6 (1991) 1745.

80. J. R. Ellis, J. S. Hagelin, D. V. Nanopoulos, K. A. Olive and M. Srednicki, Nucl. 
Phys. B 238 (1984) 453.

81. J. R. Ellis, K. A. Olive and Y. Santoso, New J. Phys. 4 (2002) 32 [arXiv:hep$\mathrm{ph} / 0202110]$.

82. R. Barbieri and A. Strumia, arXiv:hepph/0007265; R. Barbieri, A. Pomarol, R. Rattazzi and A. Strumia, arXiv:hepph/0405040.

83. M. S. Chanowitz, Phys. Rev. D 66 (2002) 073002 [arXiv:hep-ph/0207123].

84. M. Schmaltz, Nucl. Phys. Proc. Suppl. 117 (2003) 40 [arXiv:hep-ph/0210415]; JHEP 0408 (2004) 056 [arXiv:hep$\mathrm{ph} / 0407143]$.

85. T. Rizzo, talk in the 'Beyond the Standard Model' parallel session at this conference, arXiv:hep-ph/0409042; J. L. Hewett, B. Lillie and T. G. Rizzo, arXiv:hep-ph/0407059; H. Davoudiasl, J. L. Hewett, B. Lillie and T. G. Rizzo, JHEP 0405 (2004) 015 [arXiv:hep$\mathrm{ph} / 0403300]$.

86. G. W. Bennett et al. [Muon g-2 Collaboration], Phys. Rev. Lett. 92 (2004) 161802 [arXiv:hep-ex/0401008].

87. M. Davier, S. Eidelman, A. Hocker and Z. Zhang, Eur. Phys. J. C 31 (2003) 503 [arXiv:hep-ph/0308213].

88. A. Aloisio et al. [KLOE Collaboration], arXiv:hep-ex/0407048; G. Venanzoni, talk in the 'Electroweak Physics' parallel session at this conference.

89. R. R. Akhmetshin et al. [CMD-2 Collaboration], Phys. Lett. B 578 (2004) 285 [arXiv:hep-ex/0308008].

90. B. Heinemann, plenary talk at this conference.

91. I. Hinchliffe, F. E. Paige, M. D. Shapiro, J. Soderqvist and W. Yao, Phys. Rev. D 55 (1997) 5520 [arXiv:hep-ph/9610544].

92. H. Liu, plenary talk at this conference.

93. J. Ellis, N. E. Mavromatos, D. V. Nanopoulos and A. Sakharov, arXiv:grqc/0407089.

94. R. Bousso and J. Polchinski, JHEP 0006 (2000) 006 [arXiv:hep-th/0004134].
95. P. Binetruy, plenary talk at this conference.

96. N. A. Bahcall, J. P. Ostriker, S. Perlmutter and P. J. Steinhardt, Science 284 (1999) 1481 [arXiv:astro-ph/9906463]; E. Hawkins et al., Mon. Not. Roy. Astron. Soc. 346 (2003) 78 [arXiv:astroph/0212375]; M. Tegmark et al. [SDSS Collaboration], Phys. Rev. D 69 (2004) 103501 [arXiv:astro-ph/0310723].

97. S. Perlmutter et al. [Supernova Cosmology Project Collaboration], Astrophys. J. 517 (1999) 565 [arXiv:astroph/9812133]; A. G. Riess et al. [Supernova Search Team Collaboration], Astron. J. 116 (1998) 1009 [arXiv:astro$\mathrm{ph} / 9805201]$.

98. D. N. Spergel et al. [WMAP Collaboration], Astrophys. J. Suppl. 148 (2003) 175 [arXiv:astro-ph/0302209].

99. G. Barbiellini, talk in the 'Astrophysics and Cosmology' parallel session at this conference.

100. G. Ghirlanda, G. Ghisellini, D. Lazzati and C. Firmani, arXiv:astro-ph/0408350; A. S. Friedman and J. S. Bloom, arXiv:astro$\mathrm{ph} / 0408413$.

101. D. S. Akerib et al. [CDMS Collaboration], arXiv:astro-ph/0405033; P. Cushman, talk in the Astrophysics and Cosmology' parallel session at this conference.

102. A. Morselli, talk in the 'Astrophysics and Cosmology' parallel session at this conference.

103. C. Sander, talk in the "Astrophysics and Cosmology' parallel session at this conference.

104. AMANDA Collaboration, talk in the 'Astrophysics and Cosmology' parallel session at this conference.

105. J. R. Ellis, J. L. Feng, A. Ferstl, K. T. Matchev and K. A. Olive, Eur. Phys. J. C 24 (2002) 311 [arXiv:astro$\mathrm{ph} / 0110225]$. 
106. D. Miller, plenary talk at this conference.

107. K. Yokoya, plenary talk at this conference.

108. J. Brau, plenary talk at this conference.

109. J. Dorfan, plenary talk at this conference.

110. V. Lüth, plenary talk at this conference.

111. International Technology Recommendation Panel,

http://www.ligo.caltech.edu/

s skammer/ITRP Home.htm.

112. J. R. Ellis, arXiv:hep-ph/0409140.

113. J. R. Ellis, K. A. Olive, Y. Santoso and V. C. Spanos, arXiv:hep-ph/0408118.

114. M. Battaglia et al., Eur. Phys. J. C 22 (2001) 535 [arXiv:hep-ph/0106204];

B. C. Allanach et al., in Proc. of the APS/DPF/DPB Summer Study on the Future of Particle Physics (Snowmass 2001) ed. N. Graf, Eur. Phys. J. C 25 (2002) 113 [eConf C010630 (2001) P125] [arXiv:hep-ph/0202233]; M. Battaglia, A. De Roeck, J. R. Ellis, F. Gianotti, K. A. Olive and L. Pape, Eur. Phys. J. C 33 (2004) 273 [arXiv:hep-ph/0306219].

115. E. Accomando et al., [CLIC Physics Working Group], Physics at the CLIC multi-TeV linear collider, eds. M. Battaglia, A. De Roeck, J. Ellis and D. Schulte, CERN-2004-005. 